\title{
Attribute Reduction in Consistent Approximate Representation Spaces For Formal Contexts
}

\author{
Xia Wang \\ School of Mathematics, Physics and Information Science, Zhejiang Ocean University, Zhoushan, Zhejiang, China \\ E-mail: bblylm@126.com
}

\begin{abstract}
Consistent approximate representation space is a unified model for formal contexts, consistent formal decision contexts and inconsistent formal decision contexts. In this paper, notions of attribute reduction are first developed in a consistent approximate representation space, and then the verification methods of attribute reducts are proposed in a consistent approximate representation space. Finally, two methods of attribute reduction including discernibility matrix and discernibility function are given to calculate attribute reducts in a consistent approximate representation space.
\end{abstract}

Keywords- Concept lattice; Formal context; Approximate representation space; Attribute reduction

\section{INTRODUCTION}

Formal concept analysis [1,2] is an effective tool for knowledge representation, data analysis and knowledge reduction. As one of key problems in Formal concept analysis, knowledge reduction has different methods according to different purposes. In [2], Ganter et al. defined the notions of reducible objects and attributes to reduce lines and rows in a formal context. Zhang et al. [3-6] presented an approach to attribute reduction in concept lattice by finding minimal attribute sets which can keep the corresponding hierarchy of the concept lattice. In $[5,6]$, approaches to attribute reduction was also investigated in a consistent formal decision context. Then, Liu et al. studied the approaches to attribute and object reduction in attribute and object oriented concept lattices in [7]. In [8,9], Wang et al. developed a different method of attribute reduction of formal contexts, and the advantage of this approach is that it only required to preserve all extents of meet irreducible elements. In [10], attribute reduction in formal contexts was studied by preserving the granular structures in concept lattices. In [11], an efficient post-processing method was provided for pruning redundant rules basing on the properties of Galois connection. Mi et al. [12] gave a Boolean approach to computing attribute reducts in a concept lattice using discernibility function. In [13], a rule acquisition was obtained in real decision formal contexts and the corresponding method of reduction was formulated using the method of discernibility matrix and associated Boolean function. In [14], Kumar and Srinivas proposed an appraoch to reduce the size of the concept lattices based on the corresponding object-attribute matrix using fuzzy Kmeans clustering. By preserving congruence relation classes, approaches to attribute reduction in formal contexts and in consistent formal decision contexts were proposed in [15].
Wang et al. [16] studied notions and methods of attribute reduction in an inconsistent formal decision context using congruence relation classes.

In [17], consistent approximate representation spaces for formal contexts were proposed to unify the formal contexts, consistent formal decision contexts and inconsistent formal decision contexts. In this paper, we first give the definition of attribute reduction of consistent approximate representation space, which is a general definition for different formal contexts. And then two methods of attribute reduction are developed to calculate attribute reducts.

This paper first introduces preliminaries on formal concept analysis and consistent approximate representation space for formal contexts in section 2. Section 3 gives a definition of attribute reduction in a consistent approximate representation space and also obtains the verification methods of attribute reducts. And then approaches to attribute reduction are proposed in a consistent approximate representation space.

\section{PRELIMINARIES}

Some notions and properties in formal concept analysis in [2] and consistent approximate representation space for formal contexts are introduced in [17] in this section.

A. Notions and Properties about Formal Concept Analysis

Definition 1 Let $U$ and $A$ be two finite sets and $I \subset U \times A$ a relation. Then $(U, A, I)$ is called a formal context consists. Elements in $U$ are said to be objects and elements in $A$ are said to be attributes.

For any $X \subset U$ and $B \subset A$, two operators are defined as follows:

$$
\begin{gathered}
X^{\prime}=\{a \in A \mid \text { for all } x \in X,(x, a) \in I\} \\
B^{\prime}=\{x \in U \mid \text { for all } a \in B,(x, a) \in I\}
\end{gathered}
$$

Definition 2 Suppose $(U, A, I)$ is a formal context and $B \subset A$. The formal context $\left(U, B, I_{B}\right)$ is said to be a sub-context of $(U, A, I)$, when $I_{B}=I \cap(U \times B)$.

Let ${ }^{\prime B}$ represent the operator in $\left(U, B, I_{B}\right)$. Obviously, $X^{\prime B}=X^{\prime A} \cap B$ and $X^{\prime A}=X^{\prime}$ hold for any $X \subset U$. In the following text, we will use symbols $x^{\prime}$ and $a^{\prime}$ instead of $\{x\}^{\prime}$ and $\{a\}^{\prime}$ for all $(x, a) \in U \times A$.

Definition 3 For any $X \subset U$ and $B \subset A$, a pair $(X, B)$ is referred to be a formal concept of a formal context $(U, A, I)$, if $X^{\prime}=B$ and $B^{\prime}=X$. Moreover, $X$ is said to be 
the extent and $B$ the intent of the concept $(X, B)$.

The partial order of the concepts of a formal context $(U, A, I)$ is defined as $\left(X_{1}, B_{1}\right) \leq\left(X_{2}, B_{2}\right)$ which means $X_{1} \subset X_{2}$ or $B_{2} \subset B_{1}$, where $\left(X_{1}, B_{1}\right)$ and $\left(X_{2}, B_{2}\right)$ are two concepts of $(U, A, I)$. The set of all concepts of $(U, A, I)$ together with the partially order is denoted by $L(U, A, I)$. $L(U, A, I)$ is referred to be the concept lattice with respect to the formal context $(U, A, I)$. The meet and join of two concepts are given by:

$$
\begin{aligned}
& \left(X_{1}, B_{1}\right) \wedge\left(X_{2}, B_{2}\right)=\left(X_{1} \cap X_{2},\left(B_{1} \cup B_{2}\right)^{\prime \prime}\right) \\
& \left(X_{1}, B_{1}\right) \vee\left(X_{2}, B_{2}\right)=\left(\left(X_{1} \cup X_{2}\right)^{\prime \prime}, B_{1} \cap B_{2}\right) .
\end{aligned}
$$

Let $L_{U}(U, A, I)=\{X \subset U \mid(X, B) \in L(U, A, I)\}$ be the extent set of a formal context $(U, A, I)$.

Definition 4 Suppose $(U, A, I)$ and $(U, C, J)$ are two formal contexts. ( $U, A, I, C, J)$ is referred to be a formal decision context with $I \subset U \times A, J \subset U \times C$. A is called a condition attribute set and $C$ a decision attribute set respectively, and $A \cap C=\varnothing$.

\section{B. Notions and Properties about consistent approximate representation spaceconsistent}

For completeness, we introduce notions and properties of consistent approximate representation space for formal contexts.

Definition 5 Let $(U, A, I)$ be a formal context, $R=\left\{R^{a} \subset P(U) \times P(U) \mid a \in A\right\} \quad$ a family of equivalence relations on $P(U)$, and $R^{\prime}$ an equivalence relation on $P(U)$. A quadruple $\left(U, A, R, R^{\prime}\right)$ is called an approximate representation space of $(U, A, I)$.

Definition 6 Suppose $S=\left(U, A, R, R^{\prime}\right)$ is a consistent approximate representation space. For any $B \subset A$, we define $R^{B}=\underset{a \in B}{\cap} R^{a}$. If $R^{A} \subset R^{\prime}$, then $S$ is referred to be a consistent approximate representation space.

For any $B \subset A$, we denote a binary relation by

$$
R^{B}=\left\{(X, Y) \in P(U) \times P(U) \mid X^{\prime B}=Y^{\prime B}\right\} .
$$

Obviously, $R^{B}$ is an equivalence relation. We then define $\quad[X]_{R^{B}}=\left\{Y \subset U \mid(X, Y) \in R^{B}\right\} \quad$ and $C_{R^{B}}(X)=\cup\left\{Y \subset U \mid Y \in[X]_{R^{B}}\right\}$.

Lemma 1 Suppose $\left(U, A_{1}, I_{1}\right)$ and $\left(U, A_{2}, I_{2}\right)$ are two formal contexts. Then we have that $L_{U}\left(U, A_{2}, I_{2}\right) \subset L_{U}\left(U, A_{1}, I_{1}\right)$ and $\quad R^{A_{1}} \subset R^{A_{2}}$ are two equivalent conditions.

Lemma 2 Suppose $(U, A, I)$ is a formal context. Then $S=\left(U, A, R, R^{A}\right)$ is the consistent approximate representation space of the context.

Definition 7 A formal decision context $(U, A, I, C, J)$ is referred to be consistent if $R^{A} \subset R^{C}$, otherwise, it is referred to be inconsistent.
Considering Lemma 1 and Definition 7, we have that $(U, A, I, C, J)$ is consistent if and only if (iff for short) $L_{U}(U, C, J) \subset L_{U}(U, A, I)$. So we have the following result directly.

Lemma 3 Let $(U, A, I, C, J)$ be a formal decision context. Then we have that if $S=\left(U, A, R, R^{C}\right)$ is a consistent approximate representation space of $(U, A, I, C, J)$ then $(U, A, I, C, J)$ is consistent, and vice versa.

In the following text, we will construct consistent approximate representation spaces based on an inconsistent formal decision context.

Let $(U, A, I, C, J)$ be an inconsistent formal decision context. And we denote $U / R^{C}=\left\{D_{1}, D_{2}, \ldots, D_{t}\right\}$, where $D_{j}, j=1,2, \ldots, t$ is the decision congruence class. For any $X \subset U \quad, \quad B \subset A \quad$ and $\quad D_{j} \in U / R^{C} \quad$, we

define $P\left(D_{j} /[X]_{R^{B}}\right)=\frac{\left|D_{j} \cap[X]_{R^{B}}\right|}{\left|[X]_{R^{B}}\right|} \cdot \mu_{B}: P(U) \rightarrow[0,1]$ which is called a membership distribution function is defined as follows:

$$
\mu_{B}(X)=\left(P\left(D_{1} /[X]_{R^{B}}\right), \ldots, P\left(D_{t} /[X]_{R^{B}}\right)\right) .
$$

It is evident that $\mu_{B}(X)$ is a conditional probability distribution on $U / R^{C}$. For any $X \subset U$, we define the maximum decision function as

$$
\eta_{B}(X)=\left\{D_{j 0} \mid P\left(D_{j 0} /[X]_{R^{B}}\right)=\max _{1 \leq j \leq t} P\left(D_{j} /[X]_{R^{B}}\right)\right\} .
$$

And we denote

$$
\begin{aligned}
R^{\mu} & =\left\{(X, Y) \in P(U) \times P(U) \mid \mu_{A}(X)=\mu_{A}(Y)\right\}, \\
R^{\eta} & =\left\{(X, Y) \in P(U) \times P(U) \mid \eta_{A}(X)=\eta_{A}(Y)\right\} .
\end{aligned}
$$

Lemma 4 Let $(U, A, I, C, J)$ be an inconsistent formal decision context and $B \subset A$. Then $S_{\mu}=\left(U, A, R, R^{\mu}\right)$ and $S_{\eta}=\left(U, A, R, R^{\eta}\right)$ are two consistent approximate representation spaces of $(U, A, I, C, J) . S_{\mu}=\left(U, A, R, R^{\mu}\right)$ is called the distribution consistent approximate representation spaces, and $S_{\eta}=\left(U, A, R, R^{\eta}\right)$ the maximum decision consistent approximate representation spaces of $(U, A, I, C, J)$.

Remark 1 Suppose $S=\left(U, A, R, R^{\prime}\right)$ is a consistent approximate representation space. According to Lemmas 24 , if we give $R^{\prime}$ different meanings such as $R^{A}, R^{C}, R^{\mu}$ and $R^{\eta}$, then we obtain the corresponding formal context, consistent formal decision context and inconsistent formal decision context.

\section{ATTRIBUTE REDUCTION IN CONSISTENT APPROXIMATE REPRESENTATION SPACE}

In this section, we first give the notion of attribute reduction in a consistent approximate representation space and then obtain the verification methods of attribute reducts. 
Finally we propose two methods of calculating attribute reducts.

Definition 8 Let $S=\left(U, A, R, R^{\prime}\right)$ be a consistent approximate representation space. For any $B \subset A$, if $R^{B} \subset R^{\prime}$, then $B$ is referred to as an attribute consistent set of $S$. Further, for any $b \in B$ if $B-\{b\}$ is not an attribute consistent set, then $B$ is called an attribute reduct of $S$.

Theorem 1 Let $S=\left(U, A, R, R^{\prime}\right)$ be a consistent approximate representation space. Then we have the following two equivalent propositions: (I) $B$ is an attribute consistent set; (II) If $[X]_{R^{\prime}} \cap[Y]_{R^{\prime}}=\varnothing$, then $[X]_{R^{B}} \cap[Y]_{R^{B}}=\varnothing$ for any $B \subset A$, and $X, Y \subset U$.

Proof. By Definition 8, $B$ is an attribute consistent set iff $R^{B} \subset R^{\prime}$ holds. And $R^{B} \subset R^{\prime}$ is equivalent to the reslut that if $(X, Y) \notin R^{\prime}$, then $(X, Y) \notin R^{B}$ holds for any $X, Y \subset U$. That is, if $[X]_{R^{\prime}} \cap[Y]_{R^{\prime}}=\varnothing$, then $[X]_{R^{B}} \cap[Y]_{R^{B}}=\varnothing$ holds. Therefore, the result is concluded.

Definition 9 Let $S=\left(U, A, R, R^{\prime}\right)$ be a consistent approximate representation space. For any $X_{i}, X_{j} \subset U$, we denote a discernibility attribute set with respect to $\left[X_{i}\right]_{R^{A}}$ and $\left[X_{j}\right]_{R^{A}}$ by

$D_{R^{\prime}}\left(\left[X_{i}\right]_{R^{A}},\left[X_{j}\right]_{R^{A}}\right)=\left\{\begin{array}{cc}\left\{a \in A \mid\left(X_{i}, X_{j}\right) \notin R^{a}\right\},\left[X_{i}\right]_{R^{\prime}} \cap\left[X_{j}\right]_{R^{\prime}}=\varnothing \\ \varnothing, \quad\left[X_{i}\right]_{R^{\prime}} \cap\left[X_{j}\right]_{R^{\prime}} \neq \varnothing\end{array}\right.$.

And $M_{R^{\prime}}=\left(D_{R^{\prime}}\left(\left[X_{i}\right]_{R^{A}},\left[X_{j}\right]_{R^{A}}\right) \mid X_{i}, X_{j} \subset U\right)$ is referred to as a discernibility matrix with respect to $S$.

Theorem 2 Let $S=\left(U, A, R, R^{\prime}\right)$ be a consistent approximate representation space and $B \subset A$. Then we have the following two equivalent propositions: (I) $B$ is an attribute consistent set of $S$; (II) $B \cap D_{R^{\prime}}\left(\left[X_{i}\right]_{R^{A}},\left[X_{j}\right]_{R^{A}}\right) \neq \varnothing \quad$ holds for any $D_{R^{\prime}}\left(\left[X_{i}\right]_{R^{A}}\left[X_{j}\right]_{R^{A}}\right) \neq \varnothing$.

Proof. By Theorem 1 and Definition 9, we have $B$ is an attribute consistent set of $S$ iff $B$ satisfies the condition that $[X]_{R^{\prime}} \cap[Y]_{R^{\prime}}=\varnothing$ implies $[X]_{R^{B}} \cap[Y]_{R^{B}}=\varnothing$. And the condition means that if $[X]_{R^{\prime}} \cap[Y]_{R^{\prime}}=\varnothing$, then there exists $a \in B \quad$ such that $\left(X_{i}, X_{j}\right) \notin R^{a} \quad$. Therefore, $B \cap D_{R^{\prime}}\left(\left[X_{i}\right]_{R^{A}},\left[X_{j}\right]_{R^{A}}\right) \neq \varnothing \quad$ holds for any $D_{R^{\prime}}\left(\left[X_{i}\right]_{R^{A}}\left[X_{j}\right]_{R^{A}}\right) \neq \varnothing$.

Definition 10 Let $S=\left(U, A, R, R^{\prime}\right)$ be a consistent approximate representation space. $\left\{B_{i} \mid i \in \tau\right\}$ is a family of attribute subsets, where $\tau$ is an index set. We denote $C_{S}=\bigcap_{i \in \tau} B_{i} \quad N_{S}=\bigcup_{i \in l} B_{i}-C_{S}$ and $K_{S}=A-\bigcup_{i \in l} B_{i}$. Then $C_{S}, N_{S}$ and $K_{S}$ are called a core set, relatively necessary attribute set ad absolutely unnecessary attribute set respectively.

Theorem 3 Let $S=\left(U, A, R, R^{\prime}\right)$ be a consistent approximate representation space. For any $a \in A$, then we have the following three equivalent propositions: (i) $a \in C_{S}$ holds; (ii) There exist $X_{i}, X_{j} \subset U$ such that $D_{R^{\prime}}\left(\left[X_{i}\right]_{R^{A}},\left[X_{j}\right]_{R^{A}}\right)=\{a\}$; and (iii) $R^{A-\{a\}} \not \subset R^{\prime}$ holds.

Proof. Firstly, we will prove that if $a \in C_{S}$ then there exist $X_{i}, X_{j} \subset U$ such that $D_{R^{\prime}}\left(\left[X_{i}\right]_{R^{A}},\left[X_{j}\right]_{R^{A}}\right)=\{a\}$. Assume that $\forall X_{i}, X_{j} \subset U$ if $a \in D_{R^{\prime}}\left(\left[X_{i}\right]_{R^{A}},\left[X_{j}\right]_{R^{A}}\right)$, then $\left|D_{R^{\prime}}\left(\left[X_{i}\right]_{R^{A}},\left[X_{j}\right]_{R^{A}}\right)\right| \geq 2$ holds, where $\left|D_{R^{\prime}}\left(\left[X_{i}\right]_{R^{A}},\left[X_{j}\right]_{R^{A}}\right)\right|$ represents the cardinality of the set $\quad D_{R^{\prime}}\left(\left[X_{i}\right]_{R^{A}},\left[X_{j}\right]_{R^{A}}\right)$. Let $B=\left\{D_{R^{\prime}}\left(\left[X_{i}\right]_{R^{A}},\left[X_{j}\right]_{R^{A}}\right)-\{a\} \mid\left[X_{i}\right]_{R^{\prime}} \cap\left[X_{j}\right]_{R^{\prime}}=\varnothing\right\} \quad$.Then $\forall D_{R^{\prime}}\left(\left[X_{i}\right]_{R^{A}},\left[X_{j}\right]_{R^{A}}\right) \neq \varnothing \quad, \quad$ we have $B \cap D_{R^{\prime}}\left(\left[X_{i}\right]_{R^{A}},\left[X_{j}\right]_{R^{A}}\right) \neq \varnothing$. According to Theorem 2, we have $B$ is an attribute consistent set. Thus, there exists $E \subset B$ such that $E$ is an attribute reduct of $S$ and $a \notin E$, which contradict that $a \in C_{S}$. Therefore, the assumption is false. That is $a \in C_{S}$ implies that there exist $X_{i}, X_{j} \subset U$ such that $D_{R^{\prime}}\left(\left[X_{i}\right]_{R^{A}},\left[X_{j}\right]_{R^{A}}\right)=\{a\}$.

Secondly, we will prove that if there exist $X_{i}, X_{j} \subset U$ such that $D_{R^{\prime}}\left(\left[X_{i}\right]_{R^{A}},\left[X_{j}\right]_{R^{A}}\right)=\{a\}$, then $R^{A-\{a\}} \not \subset R^{\prime}$ holds. Since there exist $X_{i}, X_{j} \subset U$ such that $D_{R^{\prime}}\left(\left[X_{i}\right]_{R^{A}},\left[X_{j}\right]_{R^{A}}\right)=\{a\}$, for any $b \in A$ and $b \neq a$, the results $\quad\left[X_{i}\right]_{R^{\prime}} \cap\left[X_{j}\right]_{R^{\prime}}=\varnothing \quad, \quad\left(X_{i}, X_{j}\right) \notin R^{a} \quad$ and $\left(X_{i}, X_{j}\right) \in R^{b} \quad$ hold. Hence, $\left(X_{i}, X_{j}\right) \notin R^{\prime} \quad$ and $\left(X_{i}, X_{j}\right) \in R^{A-\{a\}}$, which implies $R^{A-\{a\}} \not \subset R^{\prime}$.

Finally, we will prove that if $R^{A-\{a\}} \not \subset R^{\prime}$, then $a \in C_{S}$ holds. Suppose $R^{A-\{a\}} \not \subset R^{\prime}$, we have that if $a \notin B$, then $B \subset A-\{a\}$ holds for any $B \subset A$. Thus, $R^{B} \not \subset R^{\prime}$. Thus, if $a \notin B$ then $B$ is not an attribute reduct of $S$. Therefore, $a \in C_{S}$ holds.

Theorem 4 Let $S=\left(U, A, R, R^{\prime}\right)$ be a consistent approximate representation space. For any $a \in A$ and $B \subset A$, we have the following two equivalent propositions: (I) $a \in K_{S} \quad$; (II) $\quad R_{S}(a) \subset R^{a} \cup R^{\prime} \quad$, where $R_{\mathrm{S}}(a)=\cup\left\{R^{B-\{a\}} \mid R^{B} \subset R^{\prime}\right\}$

Proof. Necessity. By Definition 10, if $a \in K_{S}$, then $a$ does not exist any attribute reduts of $S$. So $R^{B} \subset R^{\prime}$ implies that $R^{B-\{a\}} \subset R^{\prime}$ for any $B \subset A$. Otherwise, if $R^{B-\{a\}} \not \subset R^{\prime}$, then $R^{E} \not \subset R^{\prime}$ holds for any $E \subset B-\{a\}$. Thus, $B$ is an attribute redut of $S$ and we have the result $a \in B$, which contradict that $a \in K_{S}$. Therefore, $R_{S}(a) \subset R^{\prime} \subset\left(R^{a} \cup R^{\prime}\right)$.

Sufficiency. Suppose $R_{S}(a) \subset\left(R^{a} \cup R^{\prime}\right)$. For any $B \subset A$ we have that if $R^{B} \subset R^{\prime}$, then $R^{B-\{a\}} \subset\left(R^{a} \cup R^{\prime}\right) \quad$ holds. Hence, $\left[R^{B-\{a\}} \cap\left(R^{a}\right)^{C}\right] \subset R^{\prime}$ holds, where $\left(R^{a}\right)^{C}$ represents 
the complement of the set $R^{a}$. So, $R^{B-\{a\}}=R^{B} \cup\left[R^{B-\{a\}} \cap\left(R^{a}\right)^{C}\right] \subset R^{\prime}$ holds. That is, if $B$ is an attribute consistent set implies that $B-\{a\}$ is also an attribute consistent set for any $B \subset A$. Therefore, $a$ does not exist in any attribute reduct of $S$. The result is concluded.

Definition 11 Suppose $S=\left(U, A, R, R^{\prime}\right)$ is a consistent approximate representation space, and $M_{R^{\prime}}=\left(D_{R^{\prime}}\left(\left[X_{i}\right]_{R^{A}},\left[X_{j}\right]_{R^{A}}\right) \mid X_{i}, X_{j} \subset U\right)$ is the discernibility attribute matrix of $S$. $f\left(M_{R^{\prime}}\right)=\hat{D \in M_{R^{\prime}}, D \neq \varnothing}(\underset{a \in D}{\vee} a)$ is called a discernibility function.

Theorem 5 Let $S=\left(U, A, R, R^{\prime}\right)$ be a consistent approximate representation space and $B \subset A$. Then we have the following two equivalent propositions: (I) $B$ is an attribute reduct of $S$; (II) $\underset{a \in B}{\wedge} a$ is a prime implicant of $f\left(M_{R^{\prime}}\right)$.

Proof. Necessity. For any $B \subset A$ if $B$ is an attribute reduct of $S$, then for any $D \in M_{R^{\prime}}(D \neq \varnothing)$ implies $B \cap D \neq \varnothing$ by Theorem 2. Thus, there exists $D \in M_{R^{\prime}}(D \neq \varnothing)$ such that $B \cap D=\{a\}$ for any $a \in B$. Otherwise, $\forall D \in M_{R^{\prime}}$, if $D \neq \varnothing$, then $|B \cap D| \geq 2$ holds. Hence, $B-\{a\}$ is also an attribute consistent set of $S$ by Theorem 2, which contradicts the condition: $B$ is an attribute reduct set. The result is concluded..

Sufficiency. If $\underset{a \in B}{\wedge} a$ is a prime implicant of $f\left(M_{R^{\prime}}\right)$, then for any $a \in B$, there exists $D \in M_{R^{\prime}}(D \neq \varnothing)$ such that $B \cap D=\{a\}$. Therefore, B is an attribute consistent set of $S$, but $B-\{a\}$ is not an attribute consistent set of $S$ by Theorem 2. Thus, $B$ is an attribute reduct of $S$.

\section{ACKNOWLEDGMENT}

The authors gratefully acknowledge the support of the Natural Science Foundation of China (Nos. 61202206, 61272021, 61075120, 61173181, 11071284, 60673096 and 11071281) and the Natural Science Foundation of Zhejiang Province (No. LZ12F03002).

\section{REFERENCES}

[1] R. Wille, "Restructuring lattice theory: an approach based on hierarchies of concepts”, in: I. Rival (Ed.), Ordered Sets, Reidel, Dordrecht-Boston, 1982, pp.445--470.

[2] B. Ganter, and R. Wille, Formal Concept Analysis: Mathematical Foundations, Springer-Verlag, New York, 1999.

[3] W.X. Zhang, L. Wei, and J.J. Qi, “Attribute reduction theory and approach to concept lattice", Science in China Series F-Information Science, 48(6), 2005, pp. 713--726.

[4] W.X. Zhang, L. Wei, and J.J. Qi, "Attribute reduction in concept lattice based on discernibility matrix", Proceedings of RSFDGrC 2005, Lecture Notes in Artificial Intelligence ,2005, pp.157--165.

[5] W.X. Zhang, and G.F Qiu, Uncertain Decision Making Based on Rough Sets, Tsinghua University Publishing House, 2005.

[6] L. Wei, J.J. Qi, and W.X. Zhang, "Attribute reduction theory of concept lattice based on decision formal contexts”, Science in China Series F-Information Science, 51(7), 2008, pp. 910-923.

[7] M. Liu, M.W. Shao, W.X. Zhang, and C. Wu, "Reduction method for concept lattices based on rough set theory and its application", Computers and Mathematics with Applications, 53 , 2007, pp. 13901410.

[8] X. Wang, and J.M. Ma, "A novel approach to attribute reduction in concept lattices”, Proceedings of RSKT 2006, Lecture Notes in Artificial Intelligence 2006, pp. 522-529.

[9] X. Wang, and W.X. Zhang, "Relations of attribute reduction between object and property oriented concept lattices", Knowledge-Based Systems, 21(5), 2008, pp. 398-403.

[10] W.Z. Wu, Y. Leung and J.S. Mi, "Granular computing and knowledge reduction in formal contexts", IEEE Transactions Knowledge and Data Engineering, 2009, pp. 1461-1474.

[11] H.W. Liu, L. Liu and H.J. Zhang, "A fast pruning redundant rule method using Galois connection”, Applied Soft Computing, 11, 2011, pp. 130-137.

[12] J.S. Mi, Y. Leung and W.Z. Wu, "Approaches to attribute reduction in concept lattices induced by axialities”, Knowledge-Based Systems, 23 , 2010, pp. 504-511.

[13] J.H. Li, C.L. Mei and Y.J. Lv, "Knowledge reduction in real decision formal contexts”, Information Sciences, 189, 2012, pp. 191-207.

[14] C. A. Kumar and S. Srinivas, "Concept lattice reduction using fuzzy K-means clustering”, Expert Systems with Applications, 37 , 2010, pp. 2696-2704.

[15] X. Wang, "Approaches to attribute reduction in concept lattices based on rough set theory", International Journal of Hybrid Information Technology, 5(2), 2012, pp.67-80.

[16] X. Wang, and W. Z. Wu, "Approximate reduction in inconsistent formal decision contexts”, 2012 IEEE International Conference on Granular Computing, 2012, pp. 616-621.

[17] Y.W. Bao and X. Wang, "Consistent Approximate Representation Space for Formal Contexts", International Journal of Computer Science and Knowledge Engineering. (In press) 